\title{
Ferrimagnetism in the Mean-Field Approximation of the Mixed Spin-3/2 and Spin-5/2 Ising System
}

\author{
Hadey K. Mohamad \\ Al-Muthanna University, College of Science, Department of Physics, Samawa550, Iraq \\ E-mail address:hadeyk2002@yahoo.com
}

\begin{abstract}
Keywords: Mixed-spin Ising model; Ferrimagnet; single-ion anisotropy; phase transitions; compensation points.
\end{abstract}

\begin{abstract}
Using the Mean-field theory based on Bogoliubov inequality for the free energy, a ferrimagnetic mixed spin-3/2 and spin-5/2 Ising model with different anisotropies is investigated. The free energy of a mixed spin Ising ferrimagnetic system from MF approximation of the Hamiltonian is calculated. By minimizing the free energy, we obtain the equilibrium magnetizations and compensation points. In particular, we investigate the effect of a single-ion anisotropy on the magnetic properties including the compensation phenomenon, in order to clarify the characteristic behaviours in a series of molecular-based magnets $\mathrm{Cs}_{2} \mathrm{Mn}^{I I}\left[V^{I I}(C N)_{6}\right]$. The phase diagram of the system is also discussed in the anisotropy dependence of transition temperature. Our results of this model predict the existence of many(two or three) compensation points in the ordered system on a simple cubic lattice.
\end{abstract}

\section{Introduction:}

Recently, Several theoretical investigations have been reported concerning the extension of efforts to a mixed spin Ising model with one constituent having spin-1 and another having spin-3/2. The magnetic properties of this system have been discovered experimentally in $\left(\mathrm{Ni}_{0.22} \mathrm{Mn}_{0.60}^{\mathrm{II}} \mathrm{Fe}^{\mathrm{II}}{ }_{0.18}\right)_{1.5}\left[\mathrm{Cr}^{\mathrm{III}}(\mathrm{CN})_{6}\right] .7 .6 \mathrm{H}_{2} \mathrm{O}[1,2]$. The mean-field approximation(MFA), in spite of its limitations, is an adequate starting point[2,3]. Therefore, within the framework of MFA, it is interesting to investigate a more general mixed-spin Ising model consisting of spin-S $>1$ and spin$5 / 2$. The magnetic properties of the mixed-spin Ising system which is considered have been investigated numerically by the use of MFA, in order to clarify the physical background for the characteristic phenomena observed in the mixed ferrimagnetic models. For example, the moleculebased magnetic material $\mathrm{Cs}_{2} \mathrm{Mn}^{I I}\left[V^{I I}(\mathrm{CN})_{6}\right]$, which is prepared by the addition of manganese (II $)\left(S_{B}=5 / 2\right)$ triflate to aqueous solutions of the hexacyanovanadate $(I I)\left(S_{A}=3 / 2\right)$ ion at $0^{\circ} C[4]$.

The theoretical work may be classified into two types. In the first type, the spin compensation temperature of the $\operatorname{system}\left(T_{k}\right)$ can be obtained by requiring the total magnetization as being equal to zero for various values of anisotropies; though the reduced magnetization of the sublattices forming the system are not equal to zero[2,3,5,6,7]. In the second class of work, the first and second order phase transitions demand Landau expansion of the free energy in the order parameter $[2,5,8,9]$. In this correspondence we are concerned to work on both classes. The purpose of this work is to investigate a mixed-spin Ising model, consisting of spin-3/2 and spin-5/2. We firstly determine the ground-state phase diagram and study sublattice magnetizations of the mixed spin ferrimagnetic Ising system with various values of the anisotropies on the basis of the mean-field theory. A Landau expression of the free energy in the order parameter is demanded in this respect. It has briefly been presented the basic framework of the theory based on the Bogoliubov inequality for the free energy. The phase diagrams and sublattice magnetizations for various values of the single-ion anisotropies acting on both atoms have been discussed . 


\section{Theory:}

We start by summarizing analytical results obtained for the MFA. The central idea of the mean-field theory is very simple: we treat the nearest neighbour Ising model in zero field on a lattice containing two sublattices $A, B$ having $N$ sites, each site having $z$ nearest neighbour. The Hamiltonian of the system in Ising model, with $\sigma_{i}^{A}=3 / 2$ and $\sigma_{j}^{B}=5 / 2$, can be written as $[2,6]$ :

$$
H=-\sum_{i, j} J_{i j} \sigma_{i}^{A} \sigma_{j}^{B}-D_{A} \sum_{i}\left(\sigma_{i}^{A}\right)^{2}-D_{B} \sum_{j}\left(\sigma_{j}^{B}\right)^{2}
$$

Where the sites of sublattice $A$ are occupied by spins $\sigma_{i}^{A}$ taking the values of $\pm 1 / 2, \pm 3 / 2$, and the sites of sublattice $B$ occupied by spins $\sigma_{j}^{B}$ taking the values of $\pm 1 / 2, \pm 3 / 2, \pm 5 / 2 . D_{A}, D_{B}$ are the anisotropies acting on the spin-3/2 and spin-5/2 respectively. $J_{i j}$ is the exchange interaction between spins at sites $i$ and $j$. The expectation value of the spin variable at the sites $i$ or $j$ is given by [7]:

$$
\left\langle\sigma_{i / j}\right\rangle=\frac{1}{Z} \sum_{\left\{\sigma_{i}, \sigma_{j}\right\}} \sigma_{i / j} e^{-\beta H}
$$

where $Z$ is the partition function of the system. Here,

$$
\beta=\frac{1}{K_{B} T}
$$

A systematic way of deriving the mean-field theory for a given microscopic Hamiltonian is to start from the Bogoliubov inequality[2,8,9] :

$$
F \leq \Phi=F_{o}+\left\langle H-H_{o}\right\rangle_{o}
$$

Where $F$ is the free energy of the system, $H_{o}$ a trial Hamiltonian depending on variational parameters, $F_{o}$ the corresponding free energy , and $\langle\ldots\rangle_{o}$ denotes an average taken in the ensemble defined by $H_{o}$.

The mean-field free energy is then defined by minimizing $\Phi$ with respect to the variational parameters $\lambda_{A / B}$, that :

$$
F_{m f}=\min _{\lambda_{A / B}}\{\Phi\}
$$

This gives the best possible approximation to the true free energy for a given choice of $H_{o}$, since the inequality (Eq.6)insists that the mean-field free energy cannot fall below the true free energy. In this work we consider one of the simplest possible choices of $H_{o}$ :

$$
H_{o}=-\sum_{i}\left[\lambda_{A} \sigma_{i}^{A}+D_{A}\left(\sigma_{i}^{A}\right)^{2}\right]-\sum_{j}\left[\lambda_{B} \sigma_{j}^{B}+D_{B}\left(\sigma_{j}^{B}\right)^{2}\right]
$$

where $\lambda_{A}$ and $\lambda_{B}$ are the two variational parameters related to the two different spins respectively. Then, the approximated free energy can be obtained by substituting the Hamiltonian of the system in Eq.(2), that one has[10]: 


$$
\begin{aligned}
& f \equiv \frac{\Phi}{N}=-\frac{1}{2 \beta}\left\{\ln \left[2 e^{9 / 4 \beta D_{A}} \cosh \left(\frac{3}{2} \beta \lambda_{A}\right)+2 e^{1 / 4 \beta D_{A}} \cosh \left(\frac{1}{2} \beta \lambda_{A}\right)\right]+\right. \\
& \left.\ln \left[2 e^{25 / 4 \beta D_{B}} \cosh \left(\frac{5}{2} \beta \lambda_{B}\right)+2 e^{9 / 4 \beta D_{B}} \cosh \left(\frac{3}{2} \beta \lambda_{B}\right)+2 e^{1 / 4 \beta D_{B}} \cosh \left(\frac{1}{2} \beta \lambda_{B}\right)\right]\right\}+ \\
& 1 / 2\left(-z J m_{A} m_{B}+\lambda_{A} m_{A}+\lambda_{B} m_{B}\right)
\end{aligned}
$$

Where $N$ is the total number of sites of lattice and $z$ its nearest-neighbor coordination number. Minimizing this expression with respect to $\lambda_{A}$ and $\lambda_{B}$ gives self-consistent expressions for the mean-field as follows:

$$
\lambda_{A}=z J m_{B} \quad, \lambda_{B}=z J m_{A}
$$

with,

$$
\begin{aligned}
m_{A} \equiv\left\langle\sigma_{i}^{A}\right\rangle_{o} & =\frac{1}{2} \frac{3 \sinh \left(\frac{3}{2} \beta \lambda_{A}\right)+e^{-2 \beta D_{A}} \sinh \left(\frac{1}{2} \beta \lambda_{A}\right)}{\cosh \left(\frac{3}{2} \beta \lambda_{A}\right)+e^{-2 \beta D_{A}} \cosh \left(\frac{1}{2} \beta \lambda_{A}\right)} \\
m_{B} \equiv\left\langle\sigma_{j}^{B}\right\rangle_{o} & =\frac{1}{2} \frac{5 \sinh \left(\frac{5}{2} \beta \lambda_{B}\right)+3 e^{-4 \beta D_{B}} \sinh \left(\frac{3}{2} \beta \lambda_{B}\right)+e^{-6 \beta D_{B}} \sinh \left(\frac{1}{2} \beta \lambda_{B}\right)}{\cosh \left(\frac{5}{2} \beta \lambda_{B}\right)+e^{-4 \beta D_{B}} \cosh \left(\frac{3}{2} \beta \lambda_{B}\right)+e^{-6 \beta D_{B}} \cosh \left(\frac{1}{2} \beta \lambda_{B}\right)}
\end{aligned}
$$

To evaluate the phase diagram(second-order phase transition lines) of a mixed-spin system, one has to expand Eqs.((6),(8),(9)), that :

$$
f=f_{o}+a m_{A}^{2}+b m_{A}^{4}+\ldots
$$

where the coefficients $f_{o}$ and $a$ are given by:

$$
\begin{aligned}
& f_{o}=-\frac{1}{2 \beta} \ln \left[\left(x_{A}+y_{A}\right)\left(x_{B}+y_{B}+z_{B}\right)\right] \\
& a=-\frac{1}{2 \beta}\left[\frac{t^{2}}{8} b_{2}+\frac{t^{4}}{128} a_{1} b_{1}^{2}\right]+0.125 t z J b_{1}
\end{aligned}
$$

with,

$$
\begin{array}{lcl}
x_{A}=2 e^{9 / 4 \beta D_{A}}, & y_{A}=2 e^{1 / 4 \beta D_{A}}, \quad t=\beta z J, \\
x_{B}=2 e^{25 / 4 \beta D_{B}}, & y_{B}=2 e^{9 / 4 \beta D_{B}}, & z_{B}=2 e^{1 / 4 \beta D_{B}}, z_{B_{1}}=e^{-4 \beta D_{B}} \\
z_{B_{2}}=e^{-6 \beta D_{B}}, b_{1}=\frac{25+9 z_{B_{1}}+z_{B_{2}}}{1+z_{B_{1}}+z_{B_{2}}}, b_{2}=\frac{25 x_{B}+9 y_{B}+z_{B}}{x_{B}+y_{B}+z_{B}} \quad a_{1}=\frac{9 x_{A}+y_{A}}{x_{A}+y_{A}}
\end{array}
$$

The second-order phase transition line is then determined by $a=0$ and $b>0$. It is worth mentioning that Eq.(12) could be evaluated both Ferromagnetic phase stability limit $(J>0)$ and Ferrimagnetic one $(J<0)[2,6,8]$.

On the other hand, the ferrimagnetic case shows the signs of sublattice magnetizations are different, and there may be a compensation point, at which the total magnetization per site, that:

$$
M=\frac{1}{2}\left(m_{A}+m_{B}\right)
$$

is equal to zero, $\operatorname{but}\left(m_{A}=-m_{B} \neq 0\right)[2,9,11]$ 


\section{Results and discussions:}

Firstly, one should notice that a new behaviour not predicted in the Ne'el theory of ferrimagnetism[12], observed in our model, when a value of $D_{B} /|J|$ approaches the critical one $D_{B C} /|J|=-4.5$. For $k_{B} T /|J|=0, k_{B} T /|J|>0$, the system is passed through a first-order phase transition depending on the values of anisotropy acting on the $B$-atom. That is to say, the system is jumped at certain temperatures before being approached Curie point $[2,12]$. The first-order boundaries between different ferrimagnetic phases at low temperatures in the $\left(D_{B}, T\right)$ planes are shown in Figs.(1,2), respectively.

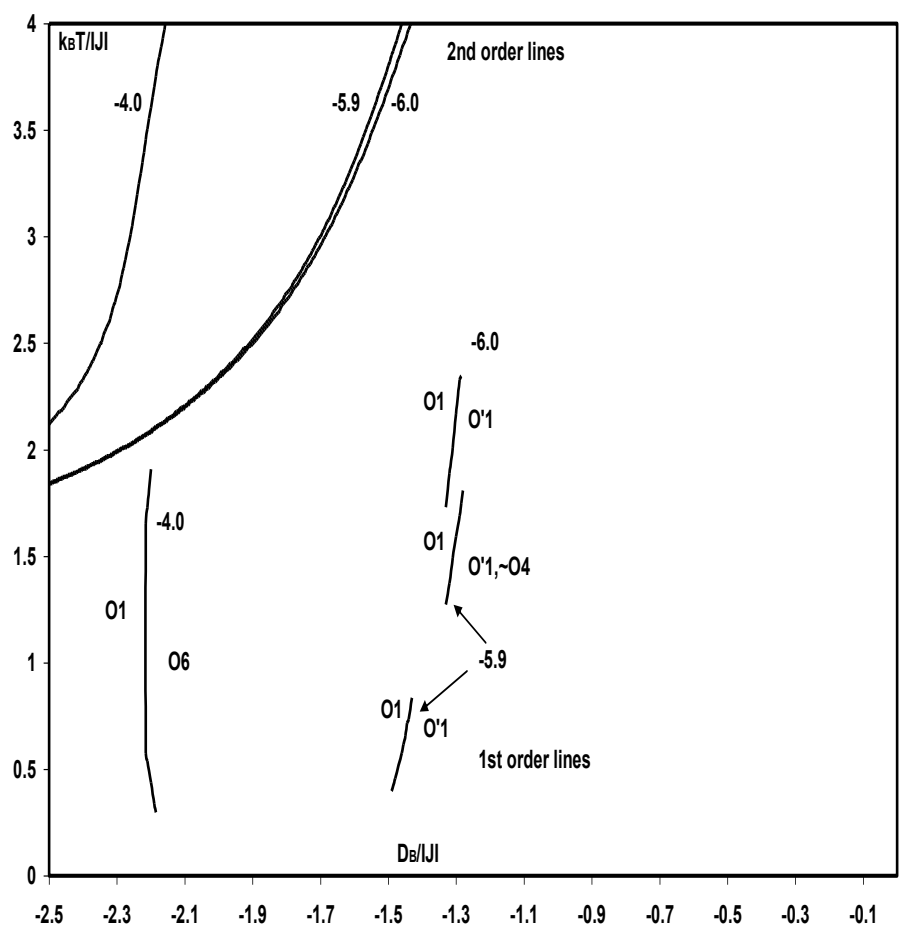

Fig.1. Low-temperature phase diagram in the $\left(D_{B}, T\right)$ plane for the mixed-spin Ising ferrimagnet with coordination number $\mathrm{z}=6$, when the value of $D_{A} / J \mid$ is changed. $O_{1}, O_{4}, O_{6}$ and $O_{1}{ }^{\prime}(1 / 2,1)$ are distinct ordered ferrimagnetic phases.

In Fig.1, the low temperature first-order transition lines are shown for three different values of $D_{A}$. In particular, the values of transition temperature in the presence of anisotropies $D_{A} /|J|=-4.0,5.9,6.0$, for fixed $D_{B} /|J|=-2.16$, are $k_{B} T_{C} /|J|=3.9709,2.1295,2.1295$, respectively.

It is worth to note the first-order phase transitions should be determined by comparing the corresponding free energies of the various solutions of Eqs.((8),(9)) for all sublattice magnetizations[2].

Now we may discuss the scale of Fig.1, in which the critical line for $D_{A} /|J|=-4.0$ shows a reentrant behavior in the phase diagram, that is to say, two transitions[13], within the range $-2.20 \leq D_{B} /|J| \leq-2.18$. It is possible within this system as we noticed to have many critical temperatures for a fixed value of $D_{A} /|J|$. Thus, it is interesting to remark that the first-order transition lines always exhibit a reentrant behaviour at low temperature[2,13,14]. 


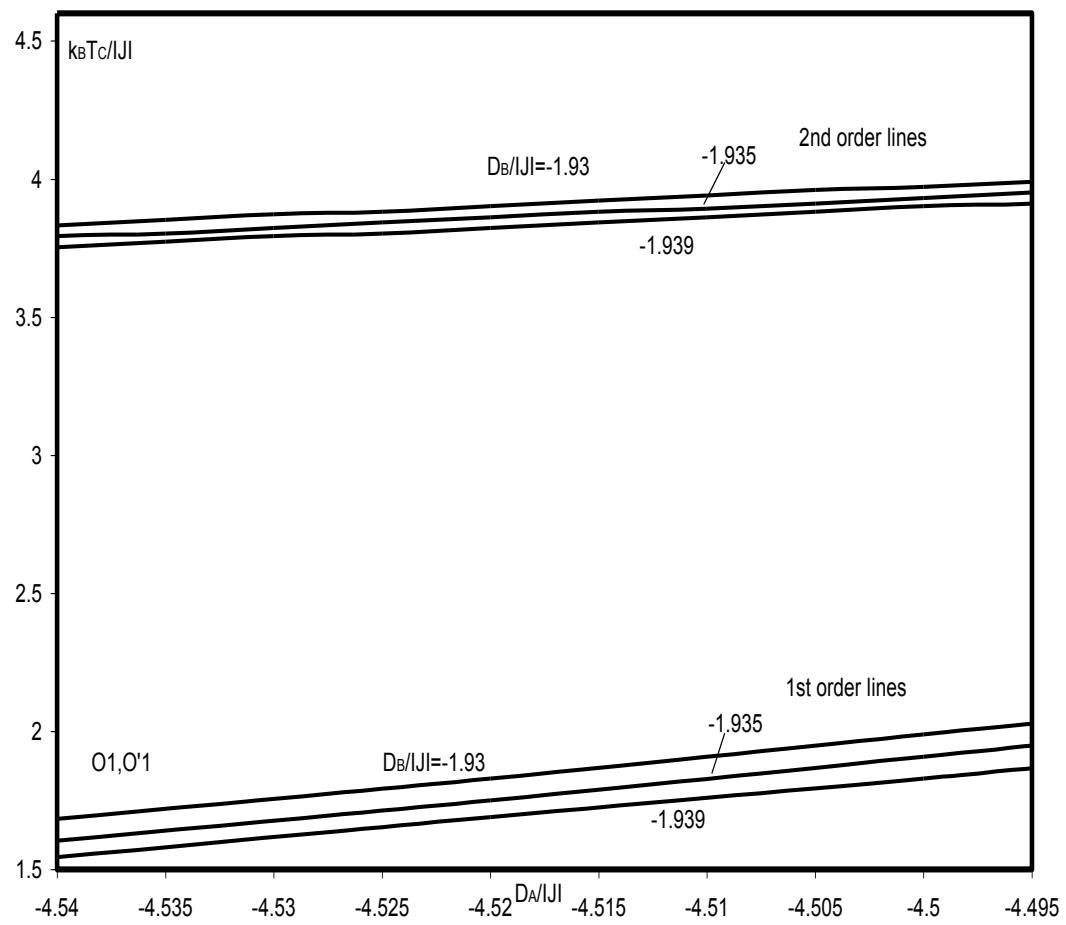

Fig.2. Low-temperature phase diagram in the $\left(D_{A}, T\right)$ plane for the mixed-spin Ising ferrimagnet with coordination number $\mathrm{z}=6$, when the value of $D_{B} /|J|$ is changed. $O_{1}$ and $O_{1}{ }^{\prime}(1 / 2,1)$ are distinct ordered ferrimagnetic phases.

On the other hand, Fig.2 shows the low temperature first-order transition lines for three different values of $D_{B}$. In particular, $D_{B} /|J|=-1.93,-1.935,-1.939$, that each first-order transition line arises from a critical point at a very low temperature and terminates at another critical point $[2,12]$. From the two Figs. $(1,2)$, we find that the first-order phase transition lines never connect to those of second-order phase transition[15,16]

Fig.3 express a close view low-temperature phase diagram for the mixed-spin Ising ferrimagnet with the coordination number $\mathrm{z}=6$, in the region of $D_{B} /|J|$ at $D_{A} /|J|=-4.0$, indicating the reentrant behavior.

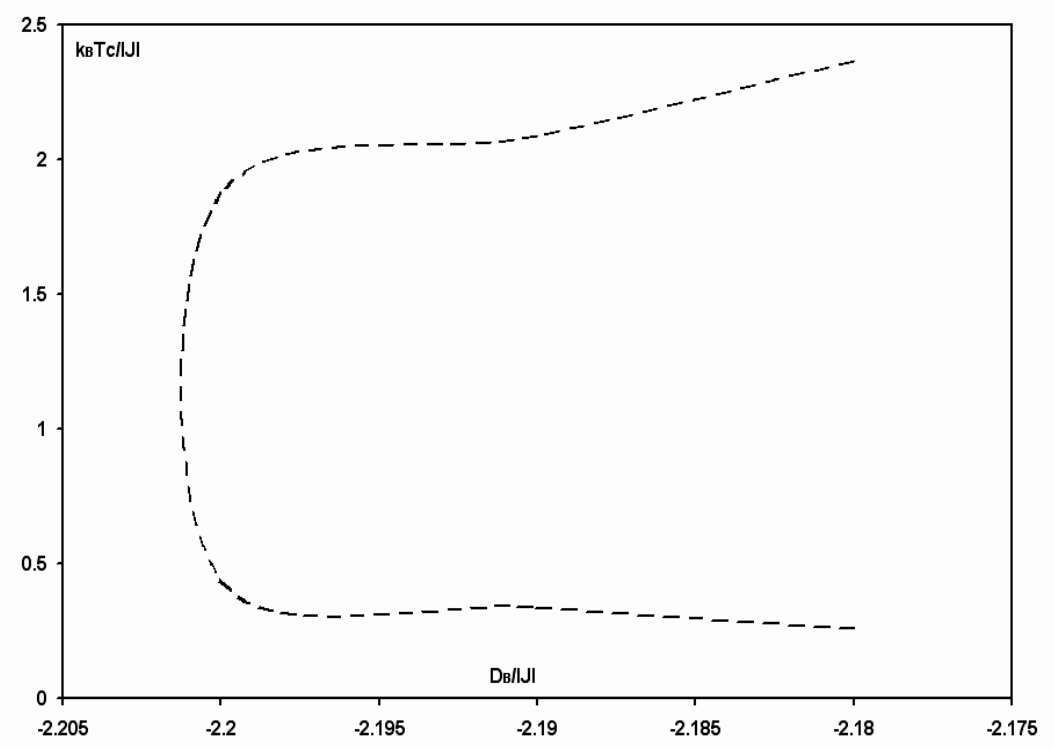

Fig.3. A close view low-temperature phase diagram in the $\left(D_{B}, \mathrm{~T}\right)$ plane for the mixed-spin Ising ferrimagnet with the coordination number $\mathrm{z}=6$, in the region of $D_{B}$ at $D_{A} /|J|=-4.0$, indicates the reentrant behavior. 
Also, it is interesting to examine the characteristic properties of the system that we find the compensation temperature $\left(T_{k}\right)$ depends strongly on the values of anisotropies $D_{A}, D_{B}$ acting on the $A$-atoms and $B$-ones, respectively. As shown in Fig.4, the system may exhibit characteristic features in the temperature dependence of the magnetization depending on the values of anisotropies $D_{B}$ of the sites occupied by atoms-B for a particular value of $D_{A}$. So, it is possible to have three compensation points at $k_{B} T /|J|=0$ and $k_{B} T /|J| \neq 0$, respectively.

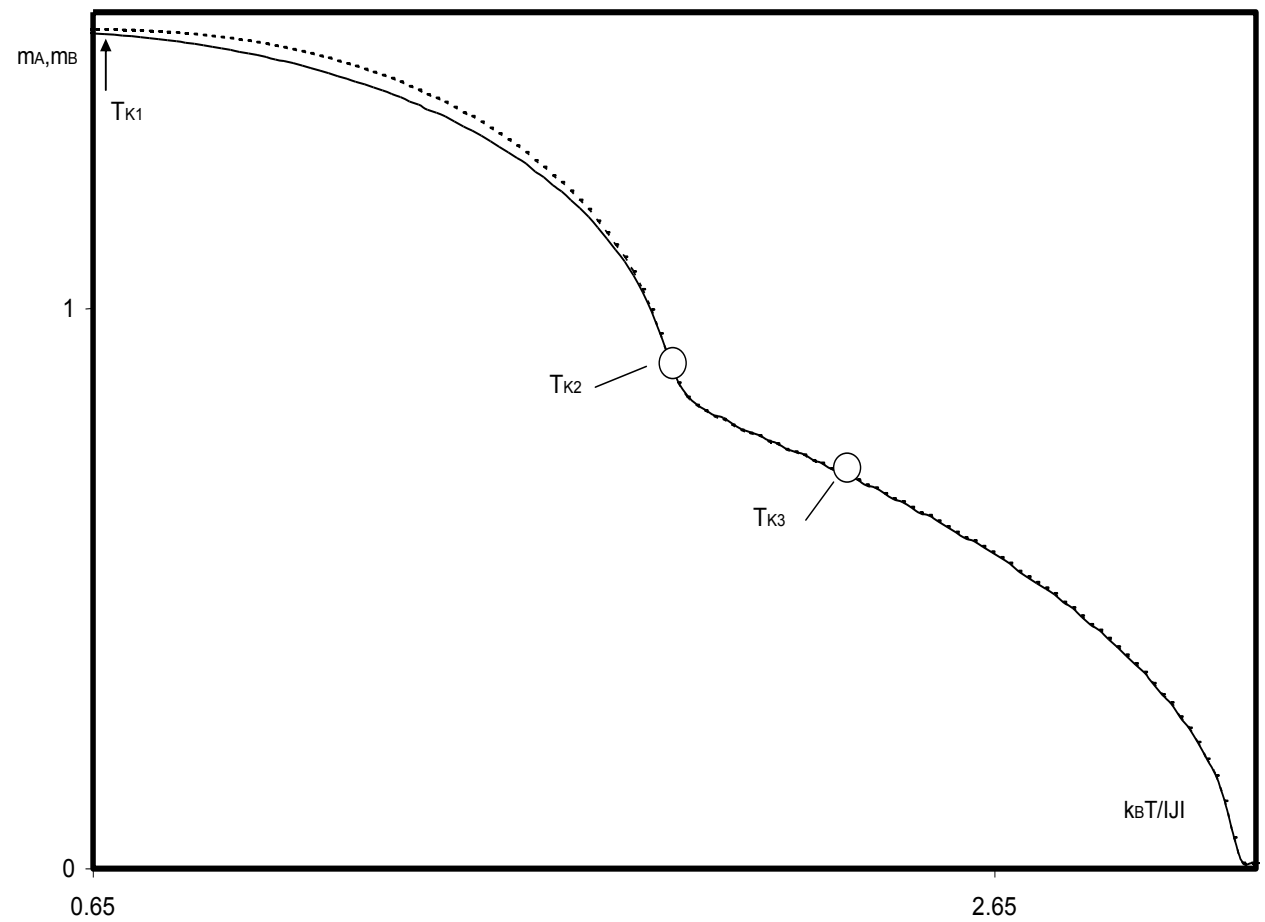

Fig.4. The temperature dependences of the sublattice magnetizations $m_{B}$ (dashed line) and $m_{A}$ (solid line) for the mixed-spin Ising ferrimagnet with the coordination number $\mathrm{z}=6$, when $D_{B} /|J|=-2.99$ and $D_{A} /|J|=-2.90 . T_{k_{1}}, T_{k_{2}}, T_{k_{3}}$ stand for the compensation temperatures.

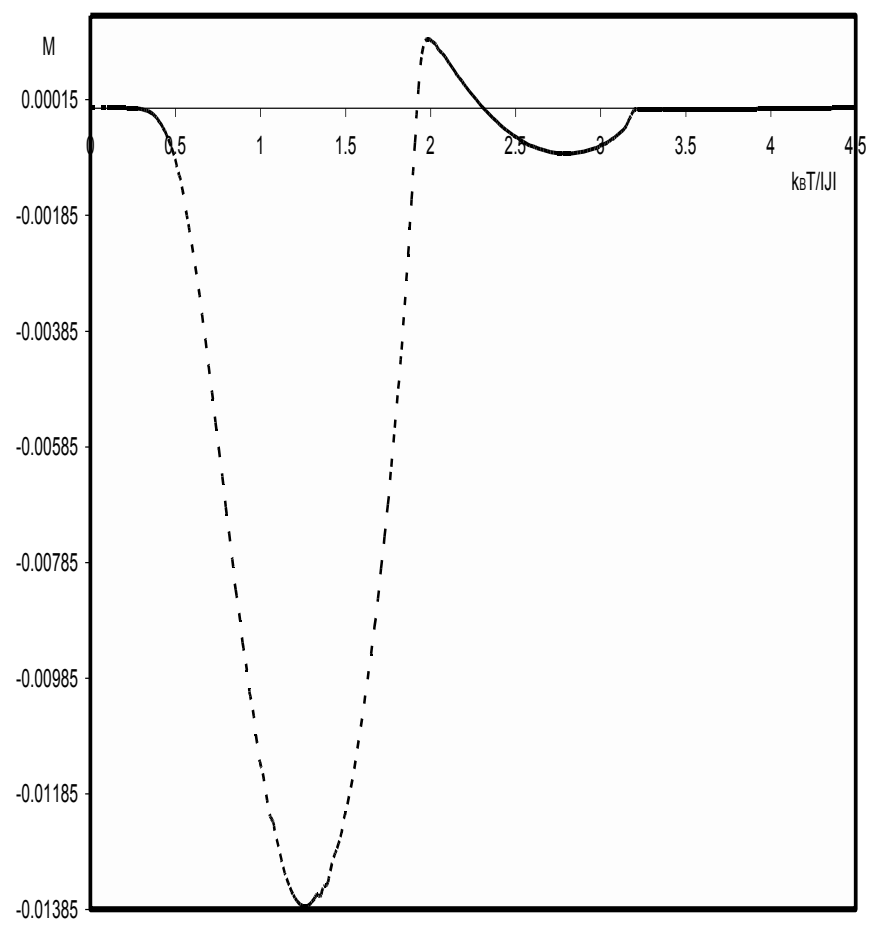

Fig.5. A close view the temperature dependences of the magnetization for the mixed-spin Ising ferrimagnet with the coordination number $\mathrm{z}=6$, when $D_{B} /|J|=-2.99$ and $D_{A} /|J|=-2.90$. 
In Figs.(4,5), we report an interesting feature of compensation temperatures for $D_{A} /|J|=-2.9$, andavalue of $D_{B} /|J|=-2.99$. So, the results showing in Fig.5 are consistent with ones derived from Fig.4. As is seen from the Figs. $(4,5)$, in the region where the system may show a compensation point, the sublattice magnetization $m_{A}$ is more ordered than the sublattice magnetization $m_{B}$ below compensation temperature. These sublattice magnetizations is still incomplete so there is a residual magnetization in the system. As the temperature is increased, at certain values of anisotropies $D_{A}, D_{B}$ acting on the A-atoms and B-atoms, respectively, the direction of this residual magnetization may switch. That is to say, due to entropy some spins can flip their directions. Thus, the sublattice magnetization $m_{B}$ becomes more ordered than the sublattice magnetization $m_{A}$ for the temperatures above compensation one. So there is an intermediate point such that the cancellation is complete[7,9]. It is worth to note that the mean-field theory for the ferrimagnetic case, predicts the existence of a compensation point in the ordered phase where the total moment vanishes[2,3].

\section{Conclusions:}

The mean-field approximation has been used in this treatment. The phase diagram of the mixed spin-3/2 and spin-5/2 Ising Ferrimagnetic system with different single-ion anisotropies has been investigated. The magnetic properties of the system with different anisotropies have been found by solving the general expressions numerically. So, the magnetization curves have exhibited some characteristics, that it has been shown the mixed-spin ferrimagnetic system is considered may exhibit an outstanding feature(many compensation points). Thus, one can compare our results with these of a mixed spin- 1 and spin-3/2 system[2], and mixed spin-3/2 and spin-5/2 on the basis of Bethe lattice[15], in which the mixed-spin model shows one or two compensation temperatures depending on the values of the crystal fields, respectively. It is worth to note that our model may exhibit three compensation points. Furthermore, a theoretical study dealing with the mixed spin-3/2 and spin-5/2 Ising model, which was treated using exact recursion relations on Cayley-tree lattices[16], that it has been found that the second-order phase transition becomes sharper when anisotropies $D_{A}$ or $D_{B}$ acting on both atoms increase. One can compare with these behaviours investigated in our model for different values of both anisotropies, as shown in Figs.(1,2). In addition to this, we have found that the first-order lines only exist for some appropriate negative values of $D_{A}$ and $D_{B}$, respectively, and furthermore the mixed-spin Ising system considered may exhibit interesting behaviours in which case a transition temperature is examined. We have observed that a transition temperature is occurred at higher one for appropriate values of $D_{A} /|J|=-2.90$ and $D_{B} /|J|=-2.99$ (see Figs.(4,5)). The researchers, using the effective-field theory for the mixed spin-3/2 and spin-5/2 Ising model on other regular planar lattices[17], have shown that the critical temperature becomes larger, as the compensation temperature becomes smaller with the increase in the interlayer coupling and the crystal-field. These results are in line with ours, when the value of $D_{B}$ is changed, for a fixed value of $D_{A}$, that one can observe Figs. $(4,5)$. Here, we can say that we find in the study of the compounds $C s_{2} \operatorname{Mn}^{I I}\left[V^{I I}(C N)_{6}\right]$, whose structure is a simple cubic lattice, a remarkable behaviour, namely the occurrence of negative magnetization at low temperature, has been investigated for the $M n^{I I} V^{I I}$ compounds, that is, to be appropriate: $\sigma_{i}^{A}=3 / 2$ for $V^{I I}$ and $\sigma_{j}^{B}=5 / 2$ for $M n^{I I}$. It is noteworthy that molecule-based magnetic material $\mathrm{Cs}_{2} \mathrm{Mn}^{I I}\left[\mathrm{~V}^{I I}(C N)_{6}\right]$ is crystallized in a face-centered cubic lattice[4]. However, as far as we are concerned that a compensation point exists or not for a such system has not yet been done, within the mean-field approximation based on the Bogoliubov inequality for the free energy. Finally, we hope that our present results may be helpful to clarify the characteristic features, for a simple cubic lattice, in a series of molecular-based magnets $C_{2} \operatorname{Mn}^{I I}\left[V^{I I}(C N)_{6}\right]$, when the experimental data of ferrimagnetic materials are analyzed . 


\section{References:}

[1]. S.Ohkoshi,Y.Abe,A.Fujishima,K.Hashimoto,Phys.Rev.Lett.82(1999)1285.

[2]. O.F.Abubrig, D.Horvath, A.Bobak, M.Jascur, Phys.A,296(2001)437.

[3]. A.Benyoussef,A.ElKenz,M.El yadari,M.J.Condens.Matter, 8,1(2007)72.

[4]. William R.Entley, Gregory S.Girolami, Science,268(1995)397.

[5]. R.Ranganathan, A.Ray, Pramana-J. Phys.,58(2002)995.

[6]. A.Bobak, Physica A,258(1998)140.

[7]. A.Dakhama, N.Benayad, J. Magn. Magn. Mater.,213(2000)117.

[8]. K.Huang, "Statistical Mechanics", $2^{\text {nd }}$ Ed., New York, 1987.

[9]. Hadey K. Mohamad,E.P.Domahsavskaya,A.F.Klinskikh,Phys.A,388(2009)4713.

[10]. Hadey K. Mohamad, International Letters of Chemistry, Physics and Astronomy, 56 (2015)31.

[11]. A.Bobak, D.Horvath, Phys.stat.sol.(b),213(1999)459.

[12]. T.Kaneyoshi, M.Jascur, P.Tomczak, J. Phys. Condens.Matter 4 (1992)L653.

[13]. J. Oitmaa, W. Zheng, Physica A 328(2003) 185.

[14]. M.Jascur,J.Strecka, Cond.Matt.phys,8(2005)869.

[15]. Erhan Albayrak, Ali Yigit, Phys.Lett.A,353(2006)121.

[16]. Rachidi A. Yessoufou, S. H. Amoussa, F. Hontinfinde, Cent.Eur.J.Phys., 7(2009)555.

[17]. Q. Zhang, G. Wei, and Y. Gu, Phys. Stat. Sol.(b) 242(2005)924. 